\title{
Separation of Low- Versus High-grade Crohn's Disease-associated Small Bowel Carcinomas is Improved by Invasive Front Prognostic Marker Analysis
}

\author{
Giovanni Arpa, a Federica Grillo,, Paolo Giuffrida, ', Gabriella Nesi,, \\ Catherine Klersy, ${ }^{e}$ Claudia Mescoli, ${ }^{f}$ Marco Vincenzo Lenti, ${ }^{\circ}$ \\ Gessica Lobascio, ${ }^{a}$ Michele Martino, ${ }^{,}$Giovanni Latella, ${ }^{9}$ Deborah Malvi, ${ }^{\text {h }}$

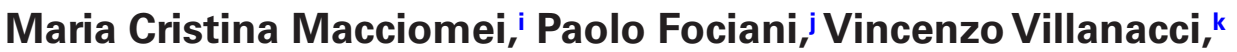 \\ Aroldo Rizzo,' Stefano Ferrero, ${ }^{\mathrm{m}}$ Fausto Sessa, ${ }^{\mathrm{n}}$ Augusto Orlandi, ${ }^{\circ}$ \\ Giovanni Monteleone, ${ }^{\mathrm{p}}$ Livia Biancone, ${ }^{\mathrm{p}}$ Laura Cantoro, ${ }^{\mathrm{q}}$ \\ Francesco Tonelli, ${ }^{r}$ Antonio Ciardi, ${ }^{\mathrm{s}}$ Gilberto Poggioli, ${ }^{\mathrm{t}}$ Fernando Rizzello, ${ }^{\mathrm{u}}$

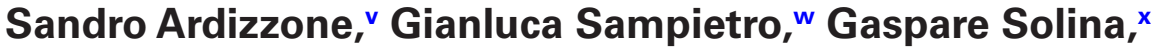 \\ Barbara Oreggia, ${ }^{y}$ Claudio Papi, ${ }^{2}$ Renata D'Incà, aa Maurizio Vecchi, ab \\ Flavio Caprioli, ab Roberto Caronna, ${ }^{\text {ac }}$ Antonietta D'Errico, ${ }^{\text {, }}$

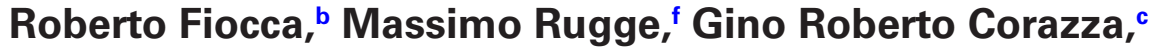 \\ Ombretta Luinetti, ${ }^{a}$ Marco Paulli, ${ }^{a}$ Enrico Solcia, ${ }^{a}$ Antonio Di Sabatino, ${ }^{c, *}$ \\ Alessandro Vanolia,*
}

\footnotetext{
aUnit of Anatomic Pathology, Department of Molecular Medicine, University of Pavia, and Fondazione IRCCS San Matteo Hospital, Pavia, Italy ${ }^{b}$ Pathology Unit, Department of Surgical and Diagnostic Sciences, University Hospital and Ospedale Policlinico San Martino IRCCS, Genova, Italy 'Department of Internal Medicine, Fondazione IRCCS San Matteo Hospital, University of Pavia, Pavia, Italy dDivision of Pathological Anatomy, Department of Surgery and Translational Medicine, University of Florence, Florence, Italy eService of Clinical Epidemiology \& Biometry, Fondazione IRCCS San Matteo Hospital, Pavia, Italy ${ }^{\mathrm{f} P a t h o l o g y}$ Unit, Department of Medicine, University of Padua, Padua, Italy ${ }^{9}$ Gastroenterology Unit, Department of Life and Environmental Sciences, University of L'Aquila, L'Aquila, Italy hDepartment of Experimental, Diagnostic and Specialty Medicine [DIMES], Institute of Oncology and Transplant Pathology, University of Bologna, Policlinico St Orsola-Malpighi Hospital, Bologna, Italy 'Pathology Unit, San Camillo-Forlanini Hospital, Rome, Italy UUnit of Pathology, Luigi Sacco University Hospital, Milan, Italy kPathology Section, Spedali Civili Hospital, Brescia, Italy 'Pathology Unit, Cervello Hospital, Palermo, Italy mDivision of Pathology, Department of Biomedical, Surgical and Dental Sciences, University of Milan, and Fondazione IRCCS Ca' Granda Ospedale Maggiore Policlinico, Milan, Italy "Department of Medicine and Surgery, University of Insubria, Varese, Italy ${ }^{\circ}$ Department of Biopathology and Image Diagnostics, University of Tor Vergata, Rome, Italy ${ }^{\mathrm{D} D e p a r t m e n t}$ of Systems Medicine, University of Tor Vergata, Rome, Italy ${ }^{9}$ Gastroenterologia Aziendale USL Umbria 1, Perugia, Italy 'Department of Surgery and Translational Medicine, University of Florence, Florence, Italy sDepartment of Radiological, Oncological, Pathological Sciences, Umberto I Hospital, La Sapienza University, Rome, Italy tSurgery of the Alimentary Tract, Department of Medical and Surgical Sciences, Sant'Orsola - Malpighi Hospital, Alma Mater Studiorum University of Bologna, Bologna, Italy "Intestinal Chronic Bowel Disease Unit, Department of Medical and Surgical Sciences, Sant'Orsola Malpighi Hospital, Alma Mater Studiorum University of Bologna, Bologna, Italy 'Gastroenterology, Luigi Sacco University Hospital, Milan, Italy w/BD Surgery, Luigi Sacco University Hospital, Milan, Italy ${ }^{\times}$General Surgery, Cervello Hospital, Palermo, Italy ${ }^{\vee}$ General Surgery Unit, Fondazione IRCCS
} 
Ca' Granda, Ospedale Policlinico, Milan, Italy zIBD, San Filippo Neri Hospital, Rome, Italy aa Gastroenterology Section, Department of Surgery, Oncology and Gastroenterology, University of Padua, Padua, Italy abGastroenterology and Endoscopy Unit, Fondazione IRCCS Ca' Granda Ospedale Maggiore Policlinico, and Department of Pathophysiology and Transplantation, University of Milan, Milan, Italy acSurgical Sciences, Umberto I Hospital, La Sapienza University, Rome, Italy

${ }^{*}$ Co-last authors

Corresponding author: Prof. Antonio Di Sabatino, MD, Clinica Medica, Fondazione IRCCS Policlinico San Matteo, Università di Pavia, Piazzale Golgi 19, 27100 Pavia, Italy. Tel.: 0382 502973; fax: 0382 502618; email: a.disabatino@smatteo.pv.it

\begin{abstract}
Background and Aims: Crohn's disease-associated small bowel carcinoma is a rare event, usually reported to have a severe prognosis. However, in previous investigations we have found a minority of cases displaying a relatively favourable behaviour, thus outlining the need to improve the histopathological prediction of Crohn's disease-associated small bowel carcinoma prognosis.

Methods: As in recent studies on colorectal cancer, a substantial improvement in prognostic evaluations has been provided by the histological analysis of the tumour invasive front; we therefore systematically analysed the tumour budding and poorly differentiated clusters in the invasive front of 47 Crohn's disease-associated small bowel carcinomas collected through the Small Bowel Cancer Italian Consortium.

Results: Both tumour budding and poorly differentiated cluster analyses proved highly effective in prognostic evaluation of Crohn's disease-associated small bowel carcinomas. In addition, they retained prognostic value when combined with two other parameters, i.e. glandular histology and stage I/II, both known to predict a relatively favourable small bowel carcinoma behaviour. In particular, association of tumour budding and poorly differentiated clusters in a combined invasive front score allowed identification of a minor subset of cancers [12/47, 25\%] characterised by combined invasive front low grade coupled with a glandular histology and a low stage [I or II] and showing no cancer-related death during a median follow-up of 73.5 months.

Conclusions: The improved distinction of lower- from higher-grade Crohn's disease-associated small bowel carcinomas provided by invasive front analysis should be of potential help in choosing appropriate therapy for these rare and frequently ominous neoplasms.
\end{abstract}

Key Words: Adenocarcinoma; grading; poorly differentiated cluster; tumour budding

\section{Introduction}

A previous study of small bowel carcinomas [SBCs] by our group found that glandular versus non-glandular histology and density of tumour-infiltrating $\mathrm{T}$ lymphocytes [TILs] were significant predictors of patient survival. ${ }^{1}$ The underlying immune-mediated predisposing disorder was also of prognostic value in SBCs; indeed, coeliac disease-associated cancers show a more favourable outcome than those associated with Crohn's disease $[\mathrm{CrD}]^{2,3}$

$\mathrm{CrD}$-associated SBC [CrD-SBC] as a whole showed poor prognosis [5-year overall survival rate: $26-38 \%],{ }^{3}$ and this is partly due to the advanced stage at diagnosis and to their often incidental finding at surgical resection for bowel stricture. The latter factor may imply suboptimal resections and/or inadequate lymph node dissection and, subsequently, the need for short-term re-intervention. ${ }^{4,5}$ However, we recently identified a smaller histological subset of CrD-SBCs, especially among those showing glandular differentiation, which was associated with less aggressive disease. ${ }^{1}$ The identification of factors which may improve the histopathological prediction of CrD-SBC prognosis is lacking, up till now.
Recent studies on colorectal and other gastrointestinal cancers have shown that careful analysis of tumour budding $[\mathrm{Tb}]$ and poorly differentiated clusters [PDCs] at the tumour invasion front may substantially improve their prognostic evaluation. ${ }^{6-13}$ The aim of this study was to evaluate these histological parameters [Tb and PDCs] on a relatively large series of CrD-SBCs collected through the Small Bowel Cancer Italian Consortium, and hopefully to improve the prognostic characterisation of this poorly known and relatively rare subset of cancers.

\section{Materials and Methods}

\subsection{Study population}

This retrospective study included 47 patients with primary non-ampullary CrD-SBCs, mostly from the ileum [ $n=44]$, two from the jejunum, and one from the duodenum. The patients, some of whom have been partly studied in previous series, ${ }^{1,2,14}$ had surgical resection and complete survival data from 15 tertiary referral Italian inflammatory bowel disease centres participating in the Small Bowel Cancer Italian Consortium. Neuroendocrine neoplasms were excluded. This study was approved by the Ethics Committee of the San Matteo Hospital Foundation of Pavia. 


\subsection{Histological evaluation}

All available haematoxylin and eosin [H\&E]-stained slides, including full-thickness sections of the tumour and encompassing the invasive front, were reviewed.

$\mathrm{Tb}$ and PDCs were analysed by two independent researchers [GA and AV] blinded to clinical and outcome data. An Eclipse Ci microscope [Nikon] with a standard 22-mm diameter eyepiece [specimen area of $0.950 \mathrm{~mm}^{2}$ under an objective lens with a magnification of $\times 20$ ] was used, and the number of buds/PDCs was divided by 1.21 to achieve the number of buds per area of $0.785 \mathrm{~mm}^{2}$ as recommended for colorectal cancer. ${ }^{9}$

\subsubsection{Definition and evaluation of $\mathrm{Tb}$}

A tumour bud is defined as a single tumour cell or a cell cluster of up to four tumour cells which develops from neoplastic glands. $\mathrm{Tb}$ was analysed along the invasive parts of the tumour using the hotspot method, which is considered to be the most useful method for assessing $\mathrm{Tb}$ in colorectal cancer. ${ }^{9}$ Initially, the invasive front of the tumour was screened using low magnification to find the areas with most Tb. For this purpose, cytokeratin 8-18 [monoclonal, clone EP17/EP30, Dako] immunohistochemistry was helpful in some challenging cases [i.e. glandular fragmentation, strong peritumoral inflammation] to allow a better visualisation of $\mathrm{Tb}$-rich areas. $\mathrm{Tb}$ was assessed from several H\&E areas, and the single field with the most budding was used for quantitation. The number of buds was counted in all cancers on H\&E staining from a single field of view using $\times 200$ total magnification [the hotspot method]. Following the International Tumour Budding Consensus Conference [ITBCC] group recommendation for colorectal cancer, we used a three-tier system: low budding [Tb1]: 0-4 buds; intermediate budding [Tb2]: 5-9 buds; and high budding [Tb3]: 10 or more buds. ${ }^{9}$

\subsubsection{Definition and evaluation of PDCs}

PDCs were defined as clusters of $\geq 5$ cancer cells that lacked a gland-like structure. The whole tumour was first scanned at low-power magnification to identify areas with the greatest number of PDCs at the invasive front. The number of PDCs in a single field of highest activity was then determined and graded as PDC1 [<5 PDCs], PDC2 [5-9 PDCs], or PDC3 [ $\geq 10$ PDCs] under an objective lens with a magnification of $\times 20 .{ }^{8,15,16}$

Cases which would have been placed into different subgroups by the two investigators had their slides re-analysed by an expert gastrointestinal pathologist [ES], and a consensus was reached. In addition, a combined invasive front $[\mathrm{CIF}]$ grade was developed as high in the presence of grade 3 for either Tb or PDCs or both and as low in the remaining cases.

The following conventional histological parameters were also investigated: tumour histotype, World Health Organization [WHO] tumour grade [for the entire tumour], and TILs and all parameters required for Tumour, Node, Metastasis [TNM] staging. ${ }^{15}$ Tumour histotype was classified as: a] glandular, b] diffuse, c] mixed [glandular plus diffuse], d] medullary, and e] non-medullary solid types, as previously described. ${ }^{1,18}$ Two cases resembling in part medullary cancers, one of which was Epstein Barr virus-positive and reinterpreted as lymphoepithelioma-like cancer, ${ }^{19}$ were omitted from this study, as recommended by Lugli et al., ${ }^{9}$ owing to technical difficulties in assessing Tb and PDC status. WHO tumour grade was based on the proportion of gland formation and categorised as grade 1 [well differentiated, $>95 \%$ ], grade 2 [moderately differentiated, $50 \%$ to $95 \%$ ], or grade 3 [poorly differentiated, $0 \%$ to $49 \%$ ]. In carcinomas with mucinous features, WHO grade, Tb, and PDCs were assessed in the area outside the mucinous component.

Table 1. Histological classification and clinico-pathological features of the 47 Crohn's disease-associated SBC cases

\begin{tabular}{|c|c|c|c|c|c|c|c|c|c|}
\hline \multirow[t]{2}{*}{ Histotype } & \multirow[t]{2}{*}{$n[\%]$} & \multirow{2}{*}{$\begin{array}{l}\text { Male sex, } \\
n[\%]\end{array}$} & \multirow{2}{*}{$\begin{array}{l}\text { Age at SBC diagnosis, } \\
\frac{\text { median }[25 \text { th- } 75 \text { th }]}{}\end{array}$} & \multirow{2}{*}{$\begin{array}{l}\text { P53 overexpression } \\
{[>50 \%]} \\
n[\%]\end{array}$} & \multirow{2}{*}{$\frac{\text { MSI/dMMR }}{n[\%]}$} & \multirow{2}{*}{$\begin{array}{l}\text { High TILs } \\
n[\%]\end{array}$} & \multicolumn{3}{|c|}{ WHO grade, $n$ [\%] } \\
\hline & & & & & & & 1 & 2 & 3 \\
\hline Glandular & 24 [51.1] & 18 [75] & 59 [54.5-69] & 14 [58.3] & $6[25]$ & 9 [37.5] & $6[25]$ & 17 [70.8] & 1 [4.2] \\
\hline Mixed & 11 [23.4] & 9 [81.8] & 56 [46-68] & $6[54.5]$ & 2 [18.2] & 2 [18.2] & 0 & 4 [36.4] & 7 [63.6] \\
\hline Diffuse & 10 [21.3] & 7 [70] & 51 [39-59] & 3 [30] & 0 & $4[40]$ & 0 & 0 & 10 [100] \\
\hline Solid & 2 [4.2] & 0 & 53 [44-62] & 2 [100] & 0 & 0 & 0 & 0 & 2 [100] \\
\hline Total & 47 [100] & 34 [72] & $57[50-68]$ & $25[53.2]$ & 8 [17] & 15 [31.9] & $6[12.8]$ & 21 [44.7] & 20 [42.5] \\
\hline
\end{tabular}

All dMMR SBCs also showed MSI by molecular analysis.

SBC, small bowel cancer; WHO, World Health Organization; dMMR, defective mismatch repair; MSI, microsatellite instability; TIL, tumour-infiltrating lymphocyte.

WHO grade distribution among histotypes: $p<0.001$.

Table 2. Classification of 47 Crohn's disease-associated SBC cases by invasive front-based grading systems

\begin{tabular}{|c|c|c|c|c|c|c|c|c|}
\hline \multirow[t]{2}{*}{ Histotype } & \multicolumn{3}{|c|}{ Tumour budding, $n$ [\%] } & \multicolumn{3}{|c|}{ Poorly differentiated clusters, $n$ [\%] } & \multicolumn{2}{|c|}{ Combined invasive front grade, $n[\%]$} \\
\hline & Tb1 & $\mathrm{Tb} 2$ & $\mathrm{~Tb} 3$ & PDC1 & PDC2 & PDC3 & Low & High \\
\hline Glandular & $12[50]$ & 4 [16.7] & 8 [33.3] & $13[54.2]$ & $6[25]$ & $5[20.8]$ & 14 [58.3] & 10 [41.7] \\
\hline Mixed & 0 & 0 & 11 [100] & $2[18.2]$ & $1[9.1]$ & $8[72.7]$ & 0 & $11[100]$ \\
\hline Diffuse & 0 & 0 & $10[100]$ & $1[10]$ & $4[40]$ & $5[50]$ & 0 & $10[100]$ \\
\hline Solid & 0 & $1[50]$ & $1[50]$ & 0 & $1[50]$ & $1[50]$ & $1[50]$ & $1[50]$ \\
\hline Total & $12[25.5]$ & $5[10.6]$ & $30[63.8]$ & $16[34]$ & 12 [25.5] & $19[40.4]$ & 15 [31.9] & 32 [68.1] \\
\hline
\end{tabular}

SBC, small bowel cancer; Tb, tumour budding; PDC, poorly differentiated clusters; CIF, combined invasive front.

$\mathrm{Tb}$ and CIF grade distribution among histotypes: $p<0.001$; PDC distribution among histotypes: $p=0.016$. 
A tumour was classified as having high TIL density when the mean number of CD3-positive TILs was > 15 per high-power field, as previously reported. ${ }^{2}$

For testing mismatch repair [MMR] protein deficiency, immunohistochemistry was performed using the standard streptavidin-biotin peroxidase procedure, with the following primary monoclonal antibodies against MLH1 [monoclonal, clone ES05, Dako], MSH2 [monoclonal, clone FE11, Dako], MSH6 [monoclonal, clone EP49, Dako], and PMS2 [monoclonal, clone EP51, Dako]. Immunostaining of MMR proteins in tumour cells was evaluated as proficient [MMRp: retained expression] or deficient [MMRd: absent expression]; only tumours showing absence of nuclear staining of all neoplastic cells in the presence of an internal positive control [intra-tumour stromal and inflammatory cells or non-tumour mucosa] were considered deficient. ${ }^{2}$ In parallel, microsatellite instability [MSI] molecular analysis was performed as previously reported. ${ }^{2}$

Finally, we searched for a correlation between invasive front markers and KRAS, NRAS, and PIK3CA mutations in $24 \mathrm{CrD}-\mathrm{SBC}$ cases, whose gene mutation analysis was available from a previous investigation, as already described. ${ }^{2}$ In addition, immunohistochemistry for p53 [monoclonal, clone DO7, Dako] was performed in all cases; a CrD-SBC was considered p53-positive when more than $50 \%$ of tumour cells showed strong nuclear p53 immunoreactivity, in line with previous studies. ${ }^{2}$

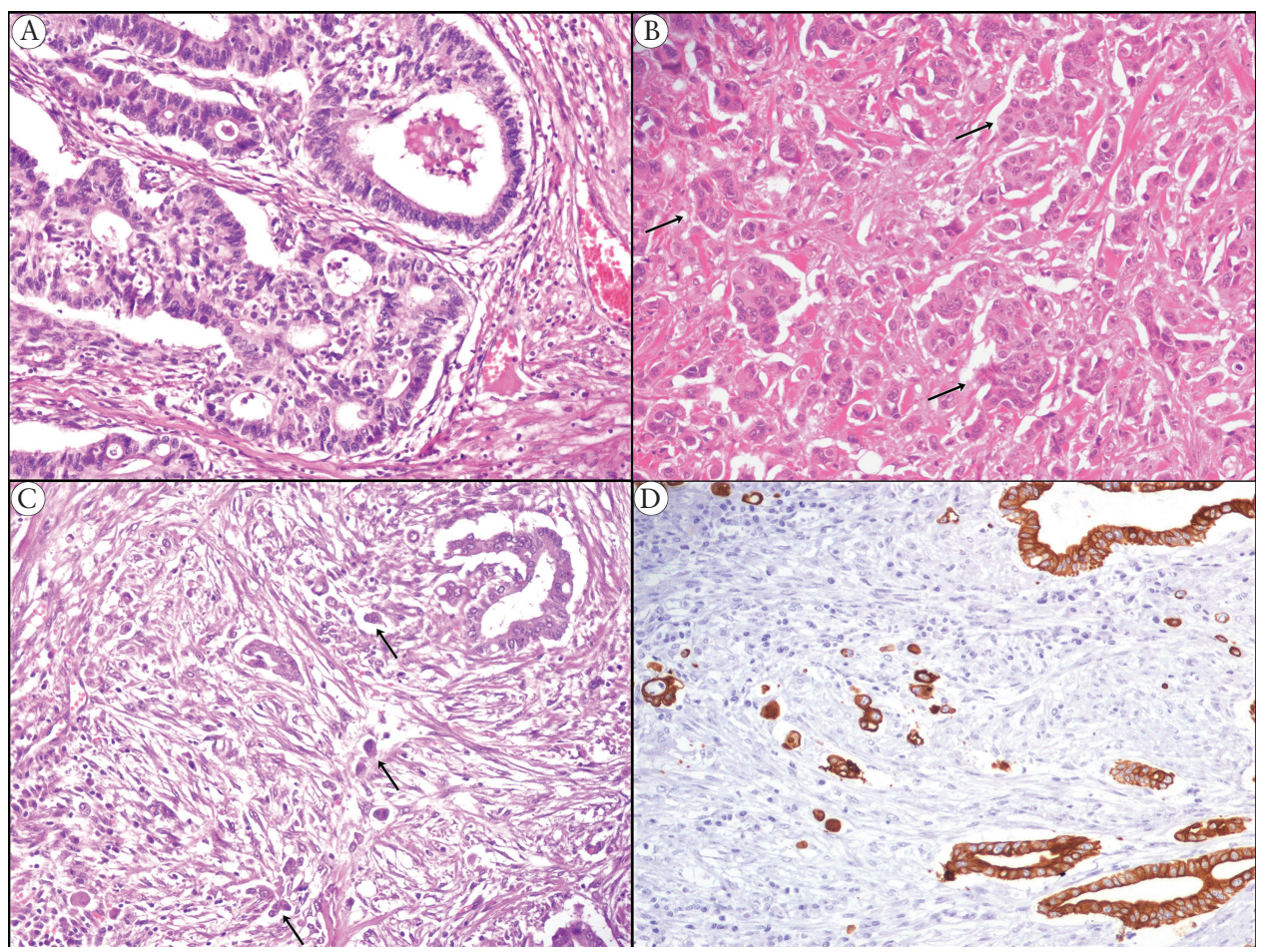

Figure 1. $[\mathrm{A}] \mathrm{A}$ glandular Crohn's disease-associated small bowel carcinoma [CrD-SBC] showing grade 1 tumour budding [Tb1] and grade 1 poorly differentiated clusters [PDC1] at the tumour invasive front [on the right] [haematoxylin and eosin; original magnification, 200x]. [B] A mixed-type CrD-SBC with grade 3 PDCs [arrows; haematoxylin and eosin; original magnification, 200x]. [C], [D] A glandular CrD-SBC showing grade 3 Tb [arrows; C, haematoxylin and eosin; D, pancytokeratin immunostaining; original magnification, 200x].

Table 3. Distribution of Tb, PDC and CIF scores among the 47 Crohn's disease-associated SBC cases classified according to pT and AJCC stage.

\begin{tabular}{|c|c|c|c|c|c|c|c|c|c|c|c|c|}
\hline \multicolumn{2}{|c|}{ Grading system } & \multirow[t]{2}{*}{ Cases, $n[\%]$} & \multicolumn{4}{|c|}{$\mathrm{pT}, n[\%]$} & \multirow[t]{2}{*}{$p$-value } & \multicolumn{4}{|c|}{ Stage, $n[\%]$} & \multirow[t]{2}{*}{$p$-value } \\
\hline & & & pT1 & $\mathrm{pT} 2$ & pT3 & pT4 & & I & II & III & IV & \\
\hline \multirow[t]{3}{*}{$\mathrm{Tb}$} & $\mathrm{Tb} 1$ & 12 [25.5] & 2 [16.7] & $4[33]$ & 5 [41.7] & $1[8.3]$ & \multirow[t]{3}{*}{0.001} & $6[50]$ & 5 [41.7] & 1 [8.33] & 0 & \multirow{3}{*}{$<0.001$} \\
\hline & $\mathrm{Tb} 2$ & 5 [10.7] & 0 & 0 & $2[40]$ & $4[60]$ & & 0 & $3[60]$ & $2[40]$ & 0 & \\
\hline & $\mathrm{Tb} 3$ & 30 [63.8] & 0 & 0 & $16[53.3]$ & 14 [46.7] & & 0 & 11 [36.7] & 13 [43.3] & $6[20]$ & \\
\hline \multirow[t]{3}{*}{ PDC } & PDC1 & $16[34.1]$ & $2[12.5]$ & $4[25]$ & 7 [43.7] & 3 [18.8] & \multirow[t]{3}{*}{0.016} & $6[37.5]$ & $6[37.5]$ & $4[25]$ & 0 & \multirow[t]{3}{*}{0.002} \\
\hline & PDC2 & $12[25.5]$ & 0 & 0 & 8 [66.7] & $4[33.3]$ & & 0 & 8 [66.7] & $2[16.7]$ & $2[16.7]$ & \\
\hline & PDC3 & $19[40.4]$ & 0 & 0 & $8[42.1]$ & 11 [57.9] & & 0 & $5[26.3]$ & 10 [52.6] & $4[21.1]$ & \\
\hline CIF & CIF low grade & 15 [31.9] & $2[13.3]$ & $4[26.7]$ & $6[40]$ & 3 [20] & \multirow[t]{2}{*}{0.002} & $6[40]$ & $8[53.3]$ & 1 [6.7] & 0 & \multirow[t]{2}{*}{$<0.001$} \\
\hline grade & CIF high grade & 32 [68.1] & 0 & 0 & $17[53.1]$ & 15 [46.9] & & 0 & $11[34.4]$ & 15 [46.9] & 6 [18.7] & \\
\hline
\end{tabular}

SBC, small bowel carcinoma; Tb, tumour budding; PDC, poorly differentiated clusters; CIF, combined invasive front; pT, extent of the tumour into the layers of the wall of the small intestine [according to the 8th edn, AJCC TNM staging system]; AJCC, American Joint Committee on Cancer; TNM, Tumour, Node, Metastasis. 


\subsection{Statistical analysis}

We used Stata 15.1 [StataCorp, College Station, TX, USA] for all computations. We considered a two-sided $p$-value $<0.05$ as statistically significant. We did not apply multiple endpoints correction for the exploratory subgroup analyses. We described data with the median and 25th-75th percentiles if continuous, and with counts and percentages if categorical. We compared them between groups with the Kruskall-Wallis test and Fisher's exact test, respectively. We computed median follow-up with the reverse Kaplan-Meier method. We analysed cancer-specific mortality by computing the mortality rate per 100 person-years, plotting the Kaplan-Meier survival curves and assessing the risk of dying for a series of candidate risk factors with the hazard ratio [HR] and $95 \%$ confidence interval [CI] derived from a Cox model. We checked the proportional hazard assumption with a test based on residuals. We computed the Harrell's c statistic for discrimination [the closer to 1 , the better, the closer to 0.5 , the worse]. Given the low number of deaths, we did not fit multivariable survival models.

\section{Results}

The histological classification of $47 \mathrm{CrD}-\mathrm{SBCs}$ and pertinent distribution of some clinico-pathological features are reported in Table 1. A general predominance of male sex and a median age of 57 years at SBC diagnosis are worth of note. Fifteen cases showed high TIL density, only eight harboured defective MMR [including seven cases with loss of MLH1/PMS2 and one case showing isolated loss of MSH6], and p53 overexpression was observed in $53.2 \%$ of CrDSBCs without significant difference among histotypes. KRAS, NRAS, and PIK3CA mutations were found in three $[12.5 \%]$, one [4\%], and

Table 4. Cancer-specific survival of 45 Crohn's disease-associated SBCs classified according to their invasion front pattern and other predictive parameters.

\begin{tabular}{|c|c|c|c|c|c|c|c|}
\hline Parameter & & Cases $n$ & Deaths $n[\%]$ & $\begin{array}{l}\text { Rate per } 100 \\
\text { person-years }[95 \% \mathrm{CI}]\end{array}$ & $\mathrm{HR}[95 \% \mathrm{CI}]$ & $\begin{array}{l}p \text {-value } \\
{[\text { Cox }]}\end{array}$ & $\begin{array}{l}\text { Harrell's c } \\
{[95 \% \mathrm{CI}]}\end{array}$ \\
\hline Tumour & Tb1 & 11 & $1[9.1]$ & 1.33 [0.19-9.44] & 1 & $<0.001$ & 0.68 [0.59-0.77] \\
\hline \multirow[t]{2}{*}{ budding } & $\mathrm{Tb} 2$ & 5 & $2[40]$ & 20.25 [5.06-80.97] & $10.6[0.95-118.7]$ & & \\
\hline & $\mathrm{Tb} 3$ & 29 & 20 [68.9] & 25.18 [16.24-39.03] & 14.72 [1.95-111.18] & & \\
\hline \multirow[t]{3}{*}{ PDC } & PDC1 & 15 & $3[20]$ & 3.69 [1.19-11.43] & 1 & 0.004 & $0.69[0.58-0.80]$ \\
\hline & PDC2 & 12 & 8 [66.7] & 23.84 [11.92-47.67] & 4.88 [1.29-18.46] & & \\
\hline & PDC3 & 18 & $12[66.7]$ & 24.23 [13.76-42.67] & $5.94[1.67-21.15]$ & & \\
\hline \multirow[t]{2}{*}{ CIF grade } & low & 14 & $2[14.3]$ & $2.45[0.61-9.78]$ & 1 & $<0.001$ & $0.66[0.57-0.75]$ \\
\hline & high & 31 & 21 [67.7] & 25.38 [16.55-38.92] & 8.27 [1.91-35.90] & & \\
\hline \multirow[t]{3}{*}{ WHO } & G1 & 5 & 0 & 0 & not evaluable $[-\infty]$ & 0.001 & $0.67[0.56-0.78]$ \\
\hline & G2 & 20 & $8[40]$ & 10.14 [5.07-20.27] & 1 & & \\
\hline & G3 & 20 & $15[75]$ & 29.97 [18.07-49.71] & $2.08[0.88-4.91]$ & & \\
\hline \multirow[t]{2}{*}{ Histotype } & glandular & 22 & 5 [22.7] & 4.59 [1.91-11.03] & 1 & $<0.001$ & $0.68[0.58-0.78]$ \\
\hline & non-glandular & 23 & 18 [78.3] & 32.38 [20.4-51.39] & $5.02[1.85-13.63]$ & & \\
\hline \multirow[t]{2}{*}{ TILs } & high & 15 & 4 [26.7] & 5.62 [2.11-14.98] & 1 & 0.027 & $0.60[0.50-0.70]$ \\
\hline & low & 30 & 19 [63.3] & 20.36 [12.99-31.92] & $3.01[1.02-8.92]$ & & \\
\hline \multirow[t]{4}{*}{ Stage } & I & 5 & 0 & 0 & not evaluable $[-\infty]$ & $<0.001$ & $0.80[0.72-0.87]$ \\
\hline & II & 19 & $6[31.6]$ & 5.81 [2.61-12.94] & 1 & & \\
\hline & III & 15 & $11[73.3]$ & 50.24 [27.82-90.72] & 8.29 [2.51-27.31] & & \\
\hline & IV & 6 & $6[100]$ & 81.39 [36.57-181.18] & $13.65[3.67-50.83]$ & & \\
\hline
\end{tabular}

SBC, small bowel cancer; Tb, tumour budding; PDC, poorly differentiated clusters; CIF, combined invasive front; TILs, tumour-infiltrating lymphocytes; HR, hazard ratio; CI: confidence interval; WHO, World Health Organization.

A

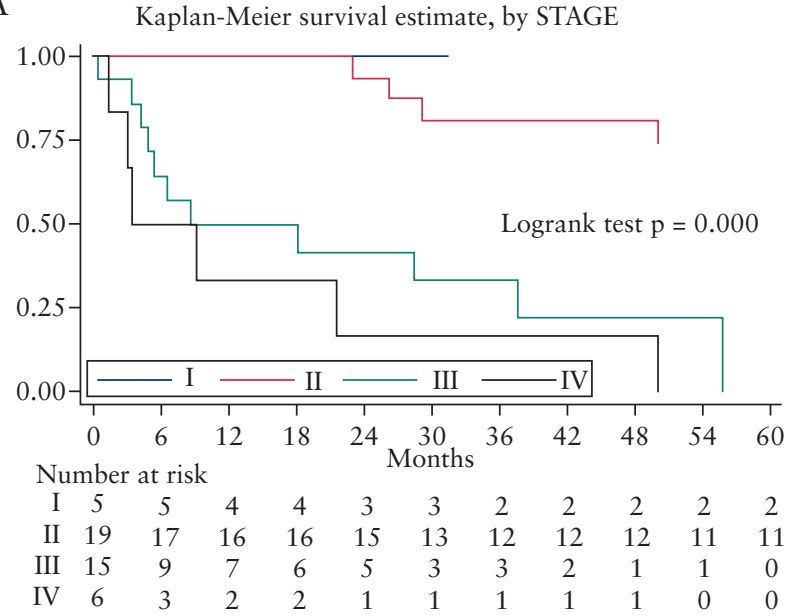

B
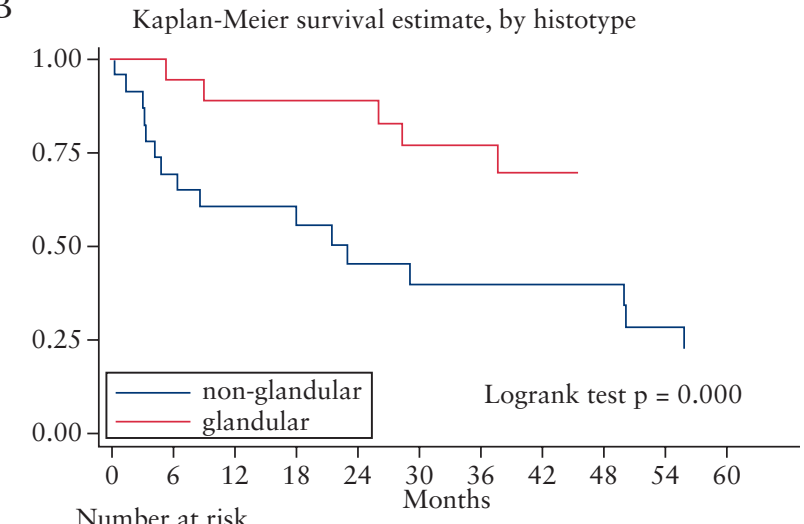

Number at risk

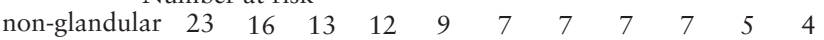

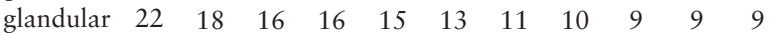

Figure 2. Kaplan-Meier survival estimates on the 45 Crohn's disease-associated small bowel carcinomas [CrD-SBCs] by stage [A] and histotype [B]. 
A Kaplan-Meier survival estimate, by $\mathrm{Tb}$

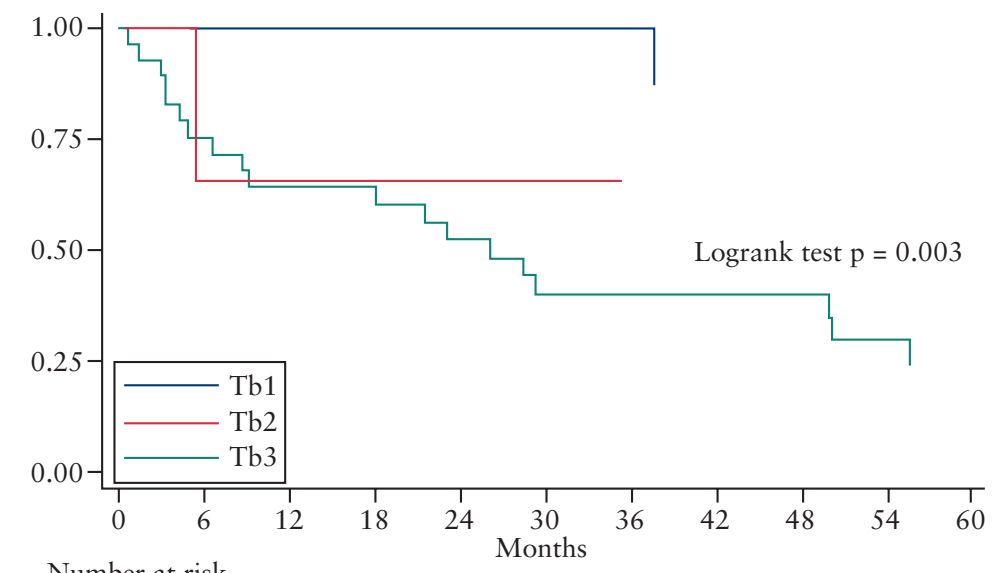

Number at risk

$\begin{array}{cccccccccccc}\text { Tb1 } & 11 & 11 & 10 & 10 & 9 & 9 & 8 & 7 & 7 & 7 & 7 \\ \text { Tb2 } & 5 & 2 & 2 & 2 & 2 & 2 & 1 & 1 & 1 & 1 & 1 \\ \text { Tb3 } & 29 & 21 & 17 & 16 & 13 & 9 & 9 & 9 & 8 & 6 & 5\end{array}$

B

Kaplan-Meier survival estimate, by PDC

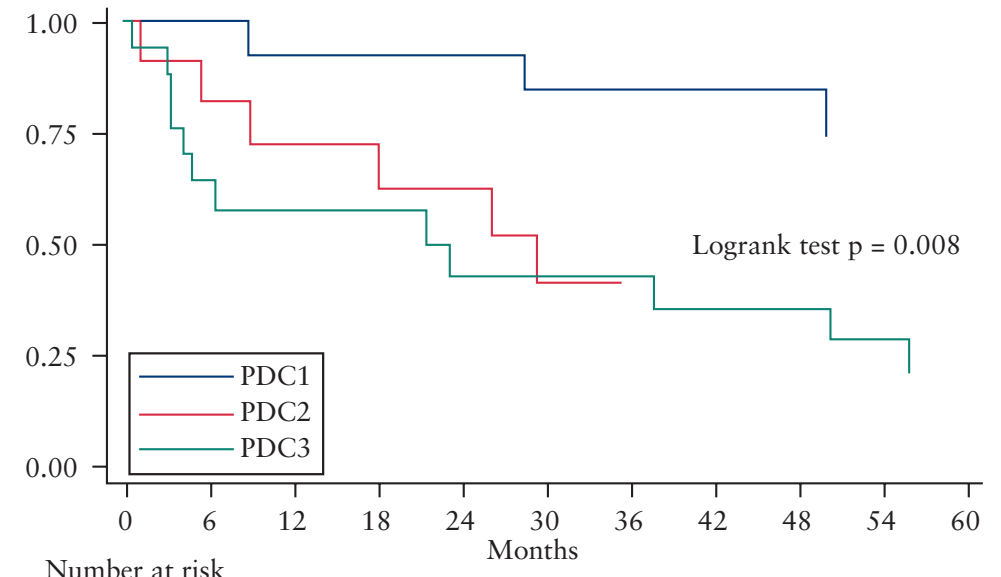

$\begin{array}{llllllllllll}\text { PDC1 } & 15 & 15 & 13 & 13 & 12 & 10 & 9 & 9 & 8 & 7 & 7\end{array}$

$\begin{array}{cccccccccccc}\text { PDC2 } & 12 & 9 & 7 & 7 & 6 & 4 & 3 & 3 & 3 & 3 & 3 \\ \text { PDC3 } & 18 & 10 & 9 & 8 & 6 & 6 & 6 & 5 & 5 & 4 & 3\end{array}$

C

Kaplan-Meier survival estimate, by CIFgrade

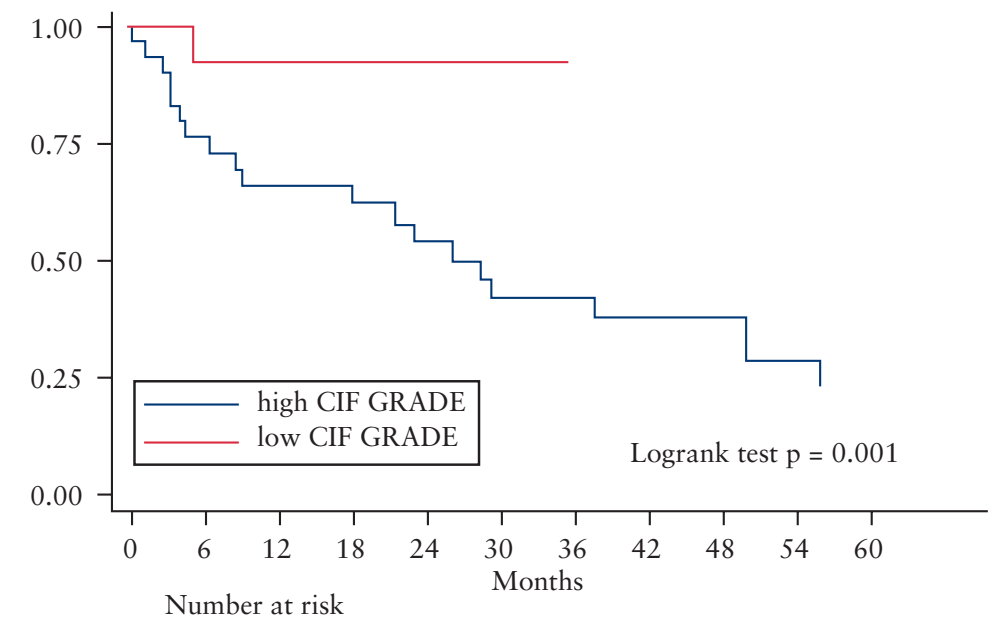

$\begin{array}{llllllllllll}\text { high CIF GRADE } & 31 & 22 & 18 & 17 & 14 & 10 & 10 & 9 & 8 & 6 & 5\end{array}$ $\begin{array}{llllllllllll}\text { low CIF GRADE } & 14 & 12 & 11 & 11 & 10 & 10 & 8 & 8 & 8 & 8 & 8\end{array}$

Figure 3. Kaplan-Meier survival estimates on the 45 Crohn's disease-associated small bowel carcinomas [CrD-SBCs] by tumour budding [A], poorly differentiated clusters $[\mathrm{B}]$, and combined invasive front grade $[\mathrm{C}]$. 
two [8\%], respectively, out of 24 cases tested. Glandular-type cancers were mostly well-to-moderately differentiated according to the WHO grading system.

The histological analysis of tumour invasion front parameters [Table 2 and Figure 1] showed a significant association $[p<0.001]$ between histotypes and either Tb or PDC grades, with a predominance of Tb1 [50\%] and PDC1 [52\%] among glandular-type cases, as well as of $\mathrm{Tb} 3[100 \%]$ and PDC3 [62\%] among diffuse/mixed cancers. As for both Tb and PDC, survival analysis gave poor separation [not significant $p$-values] of the relatively few grade 2 cases from the remaining grades, a two-tiered combined invasive front $[\mathrm{CIF}]$ grade was developed where grade 3 cases for either Tb or PDC or both defined CIF-high grade and all the remaining cases formed CIF-low grade. All diffuse and mixed tumours were placed in the CIF-high grade group, in contrast to a minority [10/24, 42\%] of glandular cases. In Table 3 , data on depth of tumour invasion [pT] and AJCC stage as a function of Tb, PDC, and CIF grade are reported: an overall correlation was found between each of the three invasive front grading systems and invasion/stage parameters. Importantly, Tb, PDC, or CIF grade were significantly associated with lymph node metastases $[p=0.001$, $p=0.023$, and $p<0.001$, respectively, with increasing rate of lymph node metastases across grades]. On the other hand, no association between invasive front markers and KRAS, NRAS, PIK3CA mutations, p53 overexpression, or MMR/MSI status was found.

Two patients died peri-operatively; the remaining 45 patients were followed up for a median of 85 [25th-75th percentiles: 31-121] months, and their cancer-specific survival data are reported in Table 4 . As expected, stage and histotype proved highly associated with survival [Figure 2], and high TILs, found in $27 \%$ of cases, and high WHO grade, with only very few grade 1 cases, were less contributive. Both $\mathrm{Tb}$ and PDC invasive front analyses gave effective patient prognostication; in particular, their combination into a CIF grade separated 14 low-grade from 31 high-grade SBC patients with highly divergent outcomes [Figure 3 and Table 4]. In addition, when CIF grade was applied to the 22 glandular histology cases [by themselves showing significantly better survival than 23 non-glandular cases], 13 CIF-low as distinguished from nine CIF-high grade tumours were identified, with a trend for divergent outcomes [HR $=6.54,95 \%$ CI: 0.73-58.6, $p=0.054]$, despite the limited number of available cases. CIF grade also gave significant results when applied to 24 stage I+II tumours, thus separating 13 low- [12 of which present in the CIF-low grade glandular group] from 11 high-grade cases, with significantly different outcomes [HR $=7.78,95 \%$ CI: 0.90-67.3, $p=0.027]$. Indeed, no cancer-related death was observed, during a median follow-up of 73.5 months, among the 12 patients with CrD-SBC showing CIF-low grade, glandular structure, and stage I or II. Of interest, six [50\%] of such tumours also showed high TILs, and three were MMRd.

\section{Discussion}

In this study of an expanded series of CrD-SBCs we confirmed the favourable prognostic influence of glandular structure [i.e. glandular histotype] in comparison with its loss to form diffuse or mixed cancerous growths, as already suggested in a previous investigation of a smaller series. ${ }^{1}$ In addition to this architectural evaluation on the whole cancer tissue, we found that selective investigation of the tumour invasive front for foci of $\mathrm{Tb}$ and/or PDCs substantially improved separation of more from less aggressive cases. Moreover, a significant association of $\mathrm{Tb}$ and PDC with lymph node metastases was found. In particular, invasive front analysis was effective, in glandular type CrD-SBCs, in identifying cases with numerous foci of cell dissociation or structural dedifferentiation [high-grade $\mathrm{Tb}$ or PDCs], which were significantly coupled with survival shortening in comparison with those with low-CIF grade [low-to-intermediate grade $\mathrm{Tb}$ and PDCs]. The use of a novel CIF two-tier grading system, which encompasses both $\mathrm{Tb}$ and PDC, renders this system easier to apply compared with the separate evaluation of $\mathrm{Tb}$ and PDC.

Our results extend to CrD-SBC the prognostic value of $\mathrm{Tb}$ and PDC evaluation, so far mainly documented for colorectal cancer [predominantly gland-forming, usual type adenocarcinoma]. Furthermore, we show that the usefulness of CIF grade is restricted to the SBC glandular subset, a finding in keeping with recent observations on gastric cancer. ${ }^{20}$ It appears that both processes involved in loss of structural differentiation, one occurring massively within the neoplasm as a whole, resulting in the diffuse and mixed tumour histotypes, and the other selectively acting at its invasive front [i.e. the high-grade $\mathrm{Tb}$ or PDCs], are strongly associated with a worse patient outcome. In other words, persistence, even at the invasive front, of the 'canonical' glandular-type structure marks a relatively less aggressive subset of CrD-SBCs, mostly non-metastatic and with a limited invasiveness.

It should be noted that loss of cellular/glandular differentiation to form 'poorly cohesive' tumours has long been recognised among gastric cancers and suggested to worsen patient prognosis. ${ }^{21-23}$ Recent molecular studies have stressed that diffuse desmoplastic cancers of the stomach, pancreas, and colon likely represent the histological counterpart of the so-called epithelial-to-mesenchymal transition operative in such cancers, with severe prognostic impact. ${ }^{24-29}$ It seems clear, especially from recent studies on colorectal carcinoma, that cancer investigation at its invasive front may capture an otherwise unapparent cancer proneness to cell dissociation and invasion, thus predicting worse postoperative behaviour. ${ }^{6-9,30}$ From our evaluation, it appears that the same phenomenon occurs in CrD-SBCs, which may add further information to stage assessment of surgical specimens.

High TIL density was associated with favourable survival, also among CrD-SBC; however, TIL assessment proved less effective in $\mathrm{CrD}-\mathrm{SBCs}$ than in coeliac disease-associated $\mathrm{SBCs},{ }^{2}$ being present in a smaller fraction of cases. TILs-rich medullary-type cancers, including the Epstein Barr virus-positive LEC with medullary-like histology, ${ }^{19}$ are known by themselves to generally display a more favourable outcome, ${ }^{31-33}$ thus overcoming the technical difficulty for $\mathrm{Tb}$ and PDC assessment reported in this rare cancer type.

In conclusion, analysis of tumour cell dissociation/ de-differentiation markers at the invasive front, such as $\mathrm{Tb}$ and PDCs, may improve the identification of highly malignant cancers and allow their separation from a minority of less aggressive neoplasms, with potential therapeutic implications. Importantly, similarly to colorectal cancer, tumour invasive front markers might help in selecting high-risk CrD-SBC patients for adjuvant chemotherapy or additional surgery. ${ }^{33-37}$

\section{Funding}

This study was partly supported by a grant from Fondazione IRCCS Policlinico San Matteo to OL. This work was also supported by a grant of the Italian Ministry of Education, University and Research [MIUR] to the Department of Molecular Medicine of the University of Pavia under the initiative 'Dipartimenti di Eccellenza [2018-2022]'.

\section{Conflict of Interest}

The authors have disclosed that they have no financial interest pertaining to this article. 


\section{Acknowledgements}

We thank all the collaborators of the Small Bowel Cancer Italian Consortium. We also thank Dr Luca Reggiani Bonetti [University of Modena and Reggio Emilia, Modena, Italy] and Prof. Valeria Barresi [University of Verona, Verona, Italy], for their invaluable support in PDC assessment training.

\section{Author Contributions}

Concept and design the study: GA, FG, ES, ADS, AV. Acquisition of data, or analysis and interpretation of data: all authors. Drafting the article: GA, FG, ES, ADS, AV. Revising the manuscript critically for important intellectual content: all authors. Final approval of the version submitted: all authors.

\section{References}

1. Vanoli A, Di Sabatino A, Martino M, et al. Small bowel carcinomas in celiac or Crohn's disease: distinctive histophenotypic, molecular and histogenetic patterns. Mod Pathol 2017;30:1453-66.

2. Vanoli A, Di Sabatino A, Furlan D, et al. Small bowel carcinomas in coeliac or Crohn's disease: clinico-pathological, molecular, and prognostic features. A study from the Small Bowel Cancer Italian Consortium. J Crobns Colitis 2017;11:942-53.

3. Giuffrida P, Vanoli A, Arpa G, et al. Small bowel carcinomas associated with immune-mediated intestinal disorders: the current knowledge. Cancers [Basel] 2018, Dec 29. doi: 10.3390/cancers11010031.

4. Sigel JE, Petras RE, Lashner BA, Fazio VW, Goldblum JR. Intestinal adenocarcinoma in Crohn's disease: a report of 30 cases with a focus on coexisting dysplasia. Am J Surg Pathol 1999;23:651-5.

5. Svrcek M, Piton G, Cosnes J, et al. Small bowel adenocarcinomas complicating Crohn's disease are associated with dysplasia: a pathological and molecular study. Inflamm Bowel Dis 2014;20:1584-92.

6. Puppa G, Senore C, Sheahan K, et al. Diagnostic reproducibility of tumour budding in colorectal cancer: a multicentre, multinational study using virtual microscopy. Histopathology 2012;61:562-75.

7. Ueno $\mathrm{H}, \mathrm{Hase} \mathrm{K}$, Hashiguchi $\mathrm{Y}$, et al. Site-specific tumor grading system in colorectal cancer: multicenter pathologic review of the value of quantifying poorly differentiated clusters. Am J Surg Pathol 2014;38:197-204.

8. Barresi V, Reggiani Bonetti L, Branca G, Di Gregorio C, Ponz de Leon M, Tuccari G. Colorectal carcinoma grading by quantifying poorly differentiated cell clusters is more reproducible and provides more robust prognostic information than conventional grading. Virchows Arch 2012;461:621-8.

9. Lugli A, Kirsch R, Ajioka Y, et al. Recommendations for reporting tumor budding in colorectal cancer based on the International Tumor Budding Consensus Conference [ITBCC] 2016. Mod Pathol 2017;30:1299-311.

10. Ohike N, Coban I, Kim GE, et al. Tumor budding as a strong prognostic indicator in invasive ampullary adenocarcinomas. Am J Surg Pathol 2010;34:1417-24.

11. Cappellesso R, Luchini C, Veronese N, et al. Tumor budding as a risk factor for nodal metastasis in pT1 colorectal cancers: a meta-analysis. Hum Pathol 2017;65:62-70.

12. Karamitopoulou E, Wartenberg M, Zlobec I, et al. Tumor budding in pancreatic cancer revisited: validation of the ITBCC scoring system. Histopathology 2018;73:137-46.

13. Dawson $\mathrm{H}$, Galuppini F, Träger $\mathrm{P}$, et al. Validation of the International Tumor Budding Consensus Conference 2016 recommendations on tumor budding in stage I-IV colorectal cancer. Hum Pathol 2019;85:145-51.

14. Vanoli A, Di Sabatino A, Martino M, et al. Epstein-Barr virus-positive ileal carcinomas associated with Crohn's disease. Virchows Arch 2017;471:549-52.

15. Ueno H, Mochizuki H, Hashiguchi $\mathrm{Y}$, et al. Risk factors for an adverse outcome in early invasive colorectal carcinoma. Gastroenterology 2004;127:385-94.
16. Konishi T, Shimada Y, Lee LH, et al. Poorly differentiated clusters predict colon cancer recurrence: an in-depth comparative analysis of invasivefront prognostic markers. Am J Surg Pathol 2018;42:705-14.

17. Amin MB, Edge SB, Greene FL, et al. AJCC Cancer Staging Manual. 8th edn. New York, NY: Springer; 2017: 221-34.

18. Chiaravalli AM, Klersy C, Vanoli A, Ferretti A, Capella C, Solcia E. Histotype-based prognostic classification of gastric cancer. World J Gastroenterol 2012;18:896-904.

19. Vanoli A, Di Sabatino A, Biancone L, et al. Small bowel Epstein-Barr virus-positive lympho-epithelioma-like carcinoma in Crohn's disease. Histopathology 2017;70:837-9.

20. Kemi N, Eskuri M, Ikäläinen J, Karttunen TJ, Kauppila JH. Tumor budding and prognosis in gastric adenocarcinoma. Am J Surg Pathol 2019;43:229-34.

21. Lauren P. The two histological main types of gastric carcinoma: diffuse and so-called intestinal-type carcinoma. An attempt at a histo-clinical classification. Acta Pathol Microbiol Scand 1965;64:31-49.

22. Carneiro F, Seixas M, Sobrinho-Simões M. New elements for an updated classification of the carcinomas of the stomach. Pathol Res Pract 1995;191:571-84.

23. Solcia E, Klersy C, Mastracci L, et al. A combined histologic and molecular approach identifies three groups of gastric cancer with different prognosis. Virchows Arch 2009;455:197-211.

24. Kim MA, Lee HS, Lee HE, Kim JH, Yang HK, Kim WH. Prognostic importance of epithelial-mesenchymal transition-related protein expression in gastric carcinoma. Histopathology 2009;54:442-51.

25. Collisson EA, Sadanandam A, Olson P, et al. Subtypes of pancreatic ductal adenocarcinoma and their differing responses to therapy. Nat Med 2011;17:500-3.

26. Wu Y, Grabsch H, Ivanova T, et al. Comprehensive genomic meta-analysis identifies intra-tumoral stroma as a predictor of survival in patients with gastric cancer. Gut 2013;62:1100-11.

27. Cancer Genome Atlas Research Network. Comprehensive molecular characterization of gastric adenocarcinoma. Nature 2014;513:202-9.

28. Cristescu R, Lee J, Nebozhyn M, et al. Molecular analysis of gastric cancer identifies subtypes associated with distinct clinical outcomes. Nat Med 2015;21:449-56.

29. Guinney J, Dienstmann R, Wang X, et al. The consensus molecular subtypes of colorectal cancer. Nat Med 2015;21:1350-6.

30. Reggiani Bonetti L, Barresi V, Bettelli S, Domati F, Palmiere C. Poorly differentiated clusters [PDC] in colorectal cancer: what is and ought to be known. Diagn Pathol 2016;11:31.

31. Minamoto T, Mai M, Watanabe $\mathrm{K}$, et al. Medullary carcinoma with lymphocytic infiltration of the stomach. Clinicopathologic study of 27 cases and immunohistochemical analysis of the subpopulations of infiltrating lymphocytes in the tumour. Cancer 1990;66:945-52.

32. Friedman K, Brodsky AS, Lu S, et al. Medullary carcinoma of the colon: a distinct morphology reveals a distinctive immunoregulatory microenvironment. Mod Pathol 2016;29:528-41.

33. Brcic I, Cathomas G, Vanoli A, Jilek K, Giuffrida P, Langner C. Medullary carcinoma of the small bowel. Histopathology 2016;69:136-40.

34. Zaanan A, Costes L, Gauthier M, et al. Chemotherapy of advanced small-bowel adenocarcinoma: a multicenter AGEO study. Ann Oncol 2010;21:1786-93.

35. Overman MJ, Kopetz S, Lin E, Abbruzzese JL, Wolff RA. Is there a role for adjuvant therapy in resected adenocarcinoma of the small intestine. Acta Oncol 2010;49:474-9.

36. Ueno H, Ishiguro M, Nakatani E, et al. Prognostic impact of tumor budding in stage II colon cancer: a prospective study [SACURA trial]. J Clin Oncol 2017;35[Suppl 15]:3609.

37. Lee VWK, Chan KF. Tumor budding and poorly-differentiated cluster in prognostication in Stage II colon cancer. Pathol Res Pract 2018;214:402-7. 